\title{
Allergie auf Nahrungsmittelzusatzstoffe selten eindeutig nachweisbar
}

\section{Farbstoffe, Konservierungsmittel und Antioxidanzien werden in zunehmendem Maße für Überempfindlichkeitsreaktionen auf Nahrungsmittel verantwortlich gemacht. Doch gelingt die Identifizierung des Auslösers nur in den seltensten Fällen.}

ei Unverträglichkeitsreaktionen auf Nahrungsmittelzusatzstoffe handelt es fast ausschließlich um pseudo-allergische Reaktionen, so T. Zuberbier von der Hautklinik der Berliner Charité. Am häufigsten sei eine Urtikaria, meist gekoppelt mit einem Angioödem. Ein Zusammenhang

\section{Allergologie '98}

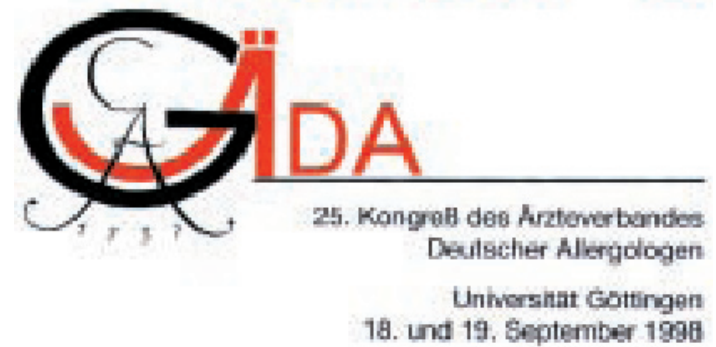
sen werden. keitsreaktionen auf Nahrungsmittelzusatzstoffe sind. Bei Kindern mit chronischer Urtikaria sei der Anteil noch höher. In einer Untersuchung hätten 12 von 16 Kinder von einer pseudoallergenarmen Diät profitiert. Bei 6 dieser Kinder wurde eine doppelblinde orale Provokationstestung durchgeführt. In 5 Fällen konnte eine eindeutige Reaktion auf Farb- und Konservierungsstoffe nachgewie-

Im allgemeinen seien die Chancen auf eine Identifizierung des Auslösers aber nicht allzu hoch. Zwar gebe es eine relativ begrenzte Liste von kaum mehr als 20 Zusatzstoffen der E-Liste, die als Auslöser bekannt sind. Da es aber 2000 bis 20000 verschiedene Nahrungsmittelzusatz- und -hilfsstoffe gebe, sei es sehr

liege besonders dann nahe, wenn es nach bestimmten Mahlzeiten spontan zu Urticae komme. Diese Patienten sprächen häufig gut auf eine allergenarme Diät an.

In einer eigenen Studie hätten $73 \%$ von 63 erwachsenen Patienten mit chronischer Urtikaria auf eine sehr strikte pseudoallergenarme Diät mit einer Besserung reagiert. 38 von 39 Patienten, die von der Diät profitiert hätten, wiesen auch 6 Monate später keine oder nur sehr geringe Symptome auf. Sie müßten allerdings weiterhin eine strenge Aufbaudiät einhalten.

Zuberbier schätzt, daß bei Erwachsenen 20\% aller Nahrungsmittelallergien in Wirklichkeit Überempfindlichwahrscheinlich, daß die Zahl der noch unentdeckten Auslöser wesentlich höher ist.

Selbst wenn der Auslöser bekannt ist, sei es für den Patienten nicht immer einfach, den Auslöser auch zu vermeiden. Die „E-Liste“ auf der Lebensmittelverpackung helfe nicht unbedingt weiter. Der Hersteller müsse dort nur jene Zusatzstoffe deklarieren, die er selber während der Herstellung zugefügt habe. Wenn er aber bereits behandelte Rohstoffe verarbeitet, erscheint unter Umständen kein Hinweis auf der Packung.

(rme)

Quelle: Vortrag von T. Zuberbier (Berlin) anläßlich des 25. Kongresses des Ärzteverbandes Deutscher Allergologen, Göttingen 1998.

\section{Migräne - niemals echte Nahrungs- mittelallergie}

Allergene in Nahrungsmitteln können keine Migräne auslösen. Für den von vielen Patienten angenommenen Zusammenhang konnte W. Aberer, Universitätshautklinik Graz, in einer Literaturrecherche keine Belege finden.

Nach den Ergebnissen wissenschaftlicher Studien betrage der Anteil der Migränepatienten, bei denen eine Allergie als unspezifischer Anfallsauslöser eine partielle oder führende Rolle spielt, nicht einmal 1\% und diese geringe Quote sei allein durch eine zufällige Häufung zu erklären.

In einem Positionspapier habe deshalb die Europäische Akademie für Allergie und klinische Immunologie unmißverständlich festgestellt, daß eine Migräne niemals Folge einer IgE-vermittelten Reaktion nach der Aufnahme von Nahrungsmitteln ist.

Eine Hauttestung sei deshalb sinnlos. Nicht dagegen eine Eliminationsdiät. Aberer empfahl, die Patienten zunächst ein Diättagebuch führen zu lassen. Wenn sich darin ein Hinweis auf eine Unverträglichkeit auf biogene Amine ergebe, was ja nicht gleichbedeutend mit einer Allergie ist, so könne eine primär unkontrollierte Elimination mancher Nahrungsmittel durchaus gerechtfertigt sein.

Aufgabe des Allergologen sei es aber auch, die Patienten vor einem Abwandern in die Alternativmedizin zu bewahren, wo sie zum Teil gesundheitsschädliche Diäten erwarten müssen. Das Ziel sei eine für den Patienten verträgliche Diät. Und für den Betroffenen sei es schließlich egal, so Aberer, ob er mit einer Typ-I-, Typ-III- oder Pseudoallergie reagiere. (rme)

Ouelle: Vortrag von W. Aberer (Graz) anläßlich des Quelle: Vortrag von W. Aberer (Graz) anläßlich des
25. Kongresses des Ärzteverbandes Deutscher Allergolo25. Kongresses des Arz 